\title{
Entropic rectification and current inversion in a pulsating channel
}

M. Florencia Carusela, and J. Miguel Rubí

Citation: The Journal of Chemical Physics 146, 184901 (2017); doi: 10.1063/1.4982884

View online: https://doi.org/10.1063/1.4982884

View Table of Contents: http://aip.scitation.org/toc/jcp/146/18

Published by the American Institute of Physics

\section{Articles you may be interested in}

Entropy facilitated active transport

The Journal of Chemical Physics 146, 185101 (2017); 10.1063/1.4982799

Model microswimmers in channels with varying cross section

The Journal of Chemical Physics 146, 174901 (2017); 10.1063/1.4981886

Directed motion from particle size oscillations inside an asymmetric channel

The Journal of Chemical Physics 146, 154103 (2017); 10.1063/1.4979984

Reexamination of multi-component non-ideal polymer solution based on the general equation for nonequilibrium reversible-irreversible coupling

The Journal of Chemical Physics 146, 184902 (2017); 10.1063/1.4982753

Communication: Mean-field theory of water-water correlations in electrolyte solutions

The Journal of Chemical Physics 146, 181103 (2017); 10.1063/1.4983221

Self-consistent generalized Langevin equation theory of the dynamics of multicomponent atomic liquids

The Journal of Chemical Physics 146, 184506 (2017); 10.1063/1.4983217

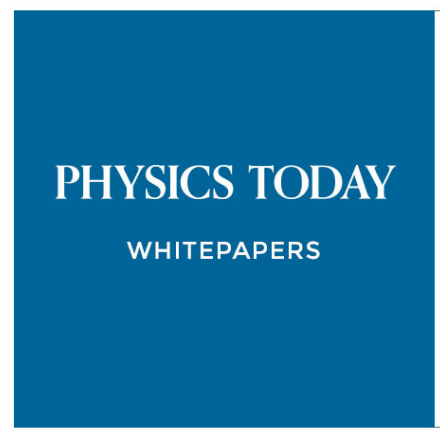

ADVANCED LIGHT CURE ADHESIVES

Take a closer look at what these environmentally friendly adhesive systems can do

\section{READ NOW}

PRESENTED BY

Q. MASTERBOND' 


\title{
Entropic rectification and current inversion in a pulsating channel
}

\author{
M. Florencia Carusela ${ }^{1, a)}$ and J. Miguel Rubi ${ }^{2}$ \\ ${ }^{1}$ Instituto de Ciencias, Universidad Nacional de General Sarmiento, J. M. Gutierrez 1150, CP 1163 Los \\ Polvorines, Buenos Aires, Argentina and CONICET, Argentina \\ ${ }^{2}$ Departament de Física Fonamental, Universitat de Barcelona, C/Martí Franquès 1, 08028 Barcelona, Spain
}

(Received 23 September 2016; accepted 20 April 2017; published online 8 May 2017)

\begin{abstract}
We show the existence of a resonant behavior of the current of Brownian particles confined in a pulsating channel. The interplay between the periodic oscillations of the shape of the channel and a force applied along its axis leads to an increase of the particle current as a function of the diffusion coefficient. A regime of current inversion is also observed for particular values of the oscillation frequency and the applied force. The model proposed is based on the Fick-Jacobs equation in which the entropic barrier and the effective diffusion coefficient depend on time. The phenomenon observed could be used to optimize transport in microfluidic devices or biological channels. Published by AIP Publishing. [http://dx.doi.org/10.1063/1.4982884]
\end{abstract}

\section{INTRODUCTION}

Transport of particles through corrugated narrow channels is frequently found in physico-chemical and biological systems in which particles are constrained to move along a main transport direction. ${ }^{1-4}$ Confinement changes significantly the transport properties of the particles and the energy conversion mechanisms. ${ }^{5-7}$ The presence of boundaries or obstacles discloses intriguing entropic contributions due to the reduction of the volume of the configuration space available to the diffusing particles. ${ }^{8}$ These entropic phenomena manifest in the appearance of entropic forces in the so-called Fick-Jacobs approximation to describe the steady-state particle densities. This equation provides a very accurate description of entropic transport in 2D and 3D channels of varying cross section. It is equivalent to a Smoluchowski equation in $1 \mathrm{D} .^{9-14}$

In many of the studies carried out up to now, channels are considered as rigid structures: kinetics of molecular motors and diffusion in zeolites, ${ }^{15-18}$ cell membrane channels, or ion translocation through protein channel. ${ }^{19-21}$ However, in many real systems, the flexible and changing nature of the channel plays a very important role in the transport properties, as in the case of vascular ${ }^{22,23}$ or peristaltic channels ${ }^{24}$ and flexible DNA nanochannels. ${ }^{25}$

Beyond the intriguing features of the transport under the effect of static boundaries, it is interesting to analyze the novel features introduced by time-changing boundaries. Rectification and resonant transport are two distinctive phenomena found in systems subject to time-dependent forces. ${ }^{26,27}$ In addition to the presence of energy barriers, confinements introduce entropic barriers that can also be time-modulated.

In recent works, the entropic transport of Brownian particles in the case of an oscillatory confined geometry was studied. A resonant transport, in the absence of an external force or when a force is acting perpendicular to the flow (e.g., a gravitational force) was found. ${ }^{28-30}$ We propose to analyze a different

\footnotetext{
a) Electronic mail: flor@ungs.edu.ar
}

situation corresponding to an external stimulus applied perpendicular to the cross section of the channel, such as gradients or fields, in the presence of a time-dependent entropic barrier. Moreover, unlike previous works, ${ }^{29,30}$ our model based on the Fick-Jacobs approximation introduces an effective diffusion coefficient also affected by the time-dependent entropic barrier. We predict not only a current rectification but also a current reversal (CR) phenomena not found when the external force is acting perpendicular to the direction of the flow. The dynamics of the particles and therefore the transport can be controlled by the diffusion coefficient, the oscillation frequency, and the strength of the force.

The article is organized as follows. In Sec. II, we introduce the entropic transport model and the effective quantities used to characterize the transport. The results obtained are presented in Sec. III. In Sec. IV, we present our main conclusions.

\section{THE MODEL}

We study the confined motion of $N$ non-interacting Brownian particles through a two dimensional periodic channel which consists of units of length $2 L$, formed by two subunits of length $L$, as is shown in Fig. 1.

The shape of the boundaries of the channel is periodically modulated in time. The height is given by:

$$
h(x, t)=\left\{\begin{array}{ll}
a_{1}(t) x^{2}+\frac{d}{2}, & 0 \leq x \leq \frac{b}{2} \\
-a_{1}(t)(x-b)^{2}+s(t), & \frac{b}{2}<x \leq b \\
-a_{2}(t)(x-b)^{2}+s(t), & \frac{b}{2}<x \leq \frac{L+b}{2} \\
a_{2}(t)(x-L)^{2}+\frac{d}{2}, & \frac{L+b}{2}<x \leq L
\end{array},\right.
$$

where $b$ indicates the location of the point of the maximum width and $d$ is the width of the bottleneck.

The time-dependent coefficients are $a_{1}(t)=\frac{2[s(t)-d]}{b^{2}}$, $a_{2}(t)=\frac{2[s(t)-d]}{(L-b)^{2}}$, and $s(t)=s_{0}+s_{1} \sin (\omega t+\Phi)$. The values of the parameters are set to guarantee asymmetric subunits. 


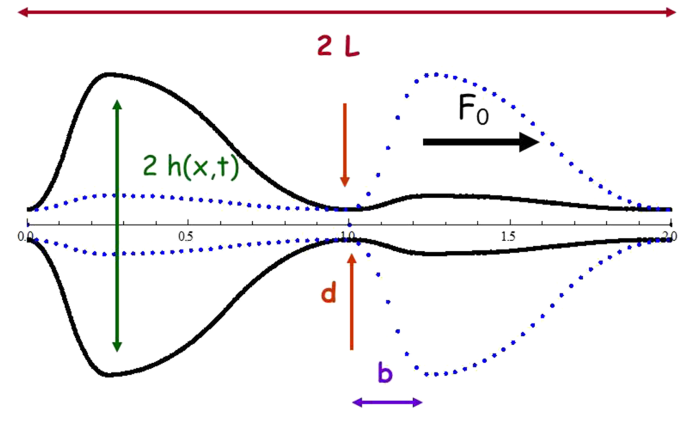

FIG. 1. Snapshot of a unit of the channel oscillating out of phase with period $T$, for two different times. The solid (black) line corresponds to $t=0$ and the dotted (blue) line corresponds to $t=T / 2$.

The phase difference between adjacent subunits inside each unit cell is given by

$$
\Phi=\left\{\begin{array}{ll}
0, & x \in[0, L] \\
{[0, \pi],} & x \in(L, 2 L]
\end{array} .\right.
$$

A phase lag $\Phi=\pi$ means that consecutive subunits can shrink and enlarge alternatively such that the total volume does not change much, a closer situation to what happens in a real transport channel.

We will analyze the transport properties by means of the Fick-Jacobs equation that govern the dynamics of the probability distribution of the ensemble of non-interacting Brownian particles

$$
\frac{\partial P(x, t)}{\partial t}=\frac{\partial}{\partial x}\left[\mathscr{D}(x, t) \frac{\partial P(x, t)}{\partial x}-\frac{\mathscr{D}(x, t)}{k_{B} T} \mathscr{F}_{\text {eff }}(x, t) P(x, t)\right] .
$$

Here $\mathscr{D}(x, t)$ is an effective diffusion coefficient that in our two dimensional case is given by ${ }^{11}$

$$
\mathscr{D}(x, t)=\frac{D}{\left(1+h^{\prime}(x, t)^{2}\right)^{(1 / 3)}},
$$

where $D$ corresponds to the diffusion coefficient and $F_{\text {eff }}(x, t)$ is an effective force acting in the x-direction due to the energy and entropic barrier contributions to the free energy $\mathscr{A}(x, t)$,

$$
\mathscr{F}_{\text {eff }}(x, t)=-\frac{\partial \mathscr{A}(x, t)}{\partial x}=F_{0}+k_{B} T \frac{h^{\prime}(x, t)}{h(x, t)}
$$

with $\mathscr{A}(x, t) \doteq E-T S=-F_{0} x-k_{B} T \ln h(x, t)$. In Fig. 2, a schematic illustration of these magnitudes is presented for a generic time.

From Eq. (3), we indentify the instantaneous particle current

$$
J(x, t)=-\left[\mathscr{D}(x, t) \frac{\partial P(x, t)}{\partial x}-\frac{\mathscr{D}(x, t)}{k_{B} T} \mathscr{F}_{\text {eff }}(x, t) P(x, t)\right] .
$$

The Fick-Jacobs approximation assumes that the probability density reaches equilibrium in the transverse direction much faster than in the longitudinal one. This requirement is fulfilled if $\left|h^{\prime}(x, t)\right|<<1$ for all times and positions, that is, when the section of the tube varies smoothly.

For the sake of simplicity, we use dimensionless quantities. We scale the lengths with the unit length $L_{o}=2 L$, times with the diffusion time $\tau_{d i f}=L_{o}^{2} \gamma /\left(k_{B} T\right)$ with $\gamma$ the Stokes'

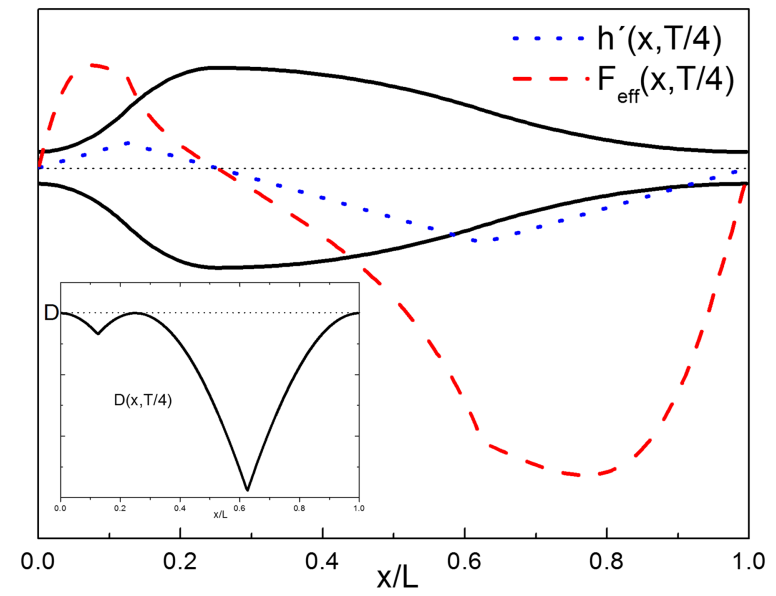

FIG. 2. Schematic illustration of $\mathscr{F}_{\text {eff }}(x, t)$ (red dashed line), $h^{\prime}(x, t)$ (blue dotted line), and $\mathscr{D}(x, t)$ (inset) in a subunit cell (black full line). Time is chosen generically as $t=T / 4$.

friction of a spherical particle of reference radius $r$, energies with $k_{B} T$, forces with units of $k_{B} T / L_{o}$, and currents with of $L_{o} / \tau_{d i f}$. Thus, the nondimensional diffusion coefficient $D$ can be expressed as the ratio between the particle size and a reference radius taken as the width of the bottleneck. A typical diffusion constant in colloids in aqueous solution is $D \approx 10^{-12} \mathrm{~m}^{2} / \mathrm{s}$. Therefore a typical Brownian time scale or average time for a particle to diffuse in a distance equal to its diameter is of the order of $1-100 \mathrm{~s}$, for particles of sizes from 1 to $10 \mu \mathrm{m}$ and velocities in the range $10^{-1}-1(\mu \mathrm{m}) / \mathrm{s} .{ }^{33,34}$ Besides, in our case, the validity of the Fick-Jacobs approach requires that the dimensionless frequency $\omega$ has to be smaller than one, this implies modulations smaller than $2 \pi 10 \mathrm{rad} / \mathrm{s}$ approximately. This range is of the order used in recent experiments on transport of molecules in confined media subject to entropic barriers and to a driving force. ${ }^{32}$

In addition to the conditions required for the validity of the Fick-Jacobs equation such as a smooth channel and slow oscillations, we assume strong viscous dynamics combined with a dilute density. Therefore under these assumptions, we can neglect hydrodynamic particle-wall and particle-particle interaction effects in the motion of the particles.

\section{NUMERICAL RESULTS}

From the dimensionless Fick-Jacobs equation, we obtain numerically the probability density $P(x, t)$ with periodic boundary conditions at $x=0$ and $x=1$.

We introduce the mean particle current as

$$
j(t)=\int_{0}^{1} J(x, t) d x
$$

with the probability current $J(x, t)$ given in Eq. (6). It can be written as ${ }^{31}$

$$
\begin{aligned}
j(t) & =\frac{d}{d t} \int_{z_{0}}^{z_{0}+1} P(x, t) x d x+J\left(z_{0}, t\right) \\
& =\frac{d}{d t}\langle x(t)\rangle+J(1, t),
\end{aligned}
$$

where $z_{0}$ is an arbitrary reference position. Here we choose $z_{0}$ $=1$ that corresponds to the upper boundary of the unit. 
Eq. (9) indicates that the particle current is composed by the motion of the "center of mass" (CM) of the ensemble and the probability current evaluated at the reference point. Then we define the position

$$
X(t) \equiv \int_{t}^{t_{0}} j\left(t^{\prime}\right) d t^{\prime}=\langle x(t)\rangle_{C M}+\int_{t}^{t_{0}} J\left(z_{0}, t^{\prime}\right) d t^{\prime}
$$

where we have defined $\left\langle x\left(t_{0}\right)\right\rangle=\left\langle x\left(t_{0}\right)\right\rangle_{C M}$.

We take the time average of the particle current in a period $T=2 \pi / \omega$,

$$
J=\frac{1}{T} \int_{t_{0}}^{T+t_{0}} j(t) d t
$$

and the time average of the mean position

$$
\bar{X}=\frac{1}{T} \int_{t_{0}}^{T+t_{0}} X(t) d t
$$

Numerical results for $\mathrm{J}$ are obtained as a function of $D$, $\omega$, and $F_{0}$. In Fig. 3 , we present a contour plot of $\mathbf{J}$ versus $D$ and the static external force $F_{0}$. We observe the presence of a current reversal (CR) for which $J$ switches from negative to positive values, even for negative values of the external force. Although $F_{0}<0$, the effect of the entropic force and the effective diffusion during a time period can compensate the negative static force. A net particle current is produced in a direction opposed to $F_{0}$ assisted by the entropic contributions. Current inversion is found for the allowed frequencies of our model, in the ranges of $D$ and $F_{0}$ shown in Fig. 3.

We observe the current as a function of $D$ when $F_{0}$ exhibits monotonic and non-monotonic behaviors. The strictly monotonic behavior is found approximately for $F_{0} \leq-1.5$ and $F_{0} \geq-0.5$. However, for intermediate values, a resonant-type regime is found where an optimal diffusion coefficient maximizes the current in an opposite direction to the force, thus yielding a most favorable situation for the coherent motion of particles in the confined geometry. In Figs. 4(a) and 4(b), we depict some representative curve levels for $\omega=0.5$ and $\omega=1.0$, respectively. This effect depends on the frequency and is enhanced as long as $\omega$ increases resulting in a greater positive current for larger $D$ as it is shown in Fig. 5. This

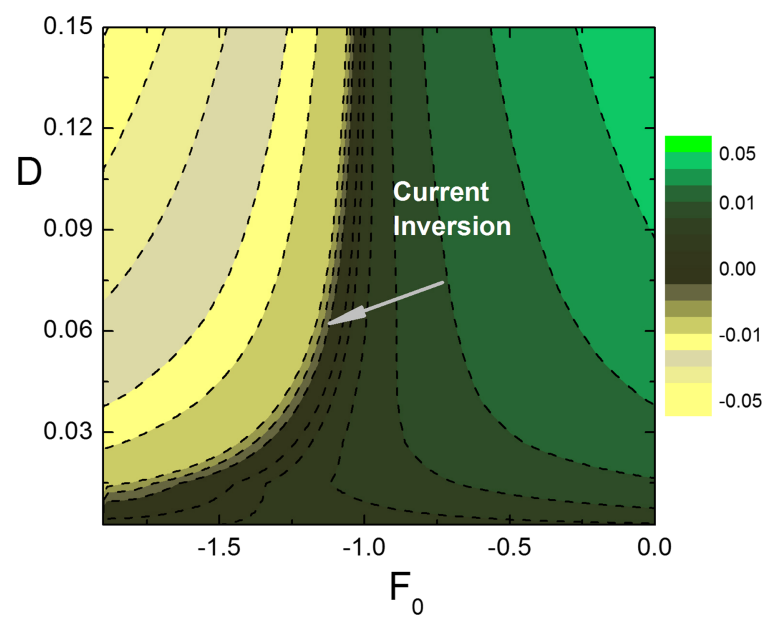

FIG. 3. Contour plot for $\mathrm{J}$ versus $D$ and $F_{0}$ for $\omega=0.5$. The values of the parameters are $s_{0}=0.45, s_{1}=0.35, b=0.25, d=0.15, L=1$. Currents are given in units of $\mu \mathrm{m} / \mathrm{s}$.
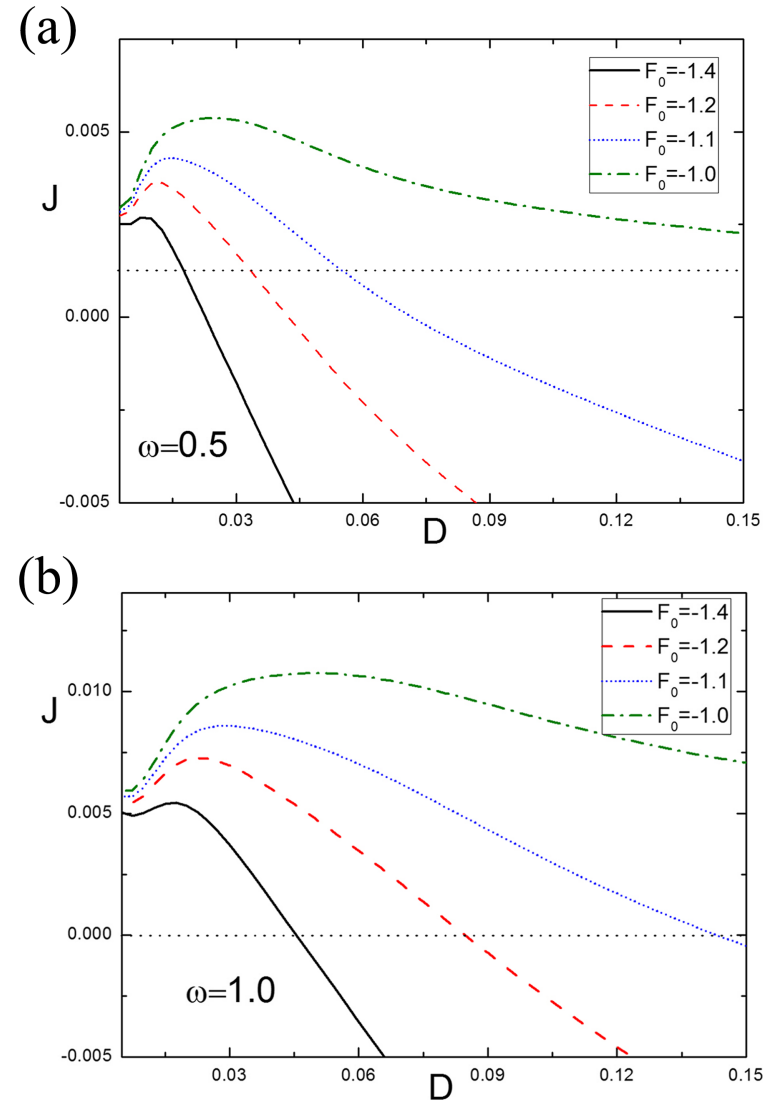

FIG. 4. J versus $D$ for different values of $F_{0}$ indicated in the figures. Panel (a) corresponds to $\omega=0.5$ and (b) to $\omega=1$. Other parameters as in Fig. 3 .

resonant-type response is appreciable for faster oscillations; in the limit of a static channel $(\omega \rightarrow 0)$, the effect is washed up. Moreover when $\omega$ increases, not only the maximum but also the crossings to negative currents (CR) move to larger values of $D$ as it is also shown in Fig. 4.

The dependence on $D$ reflects the dependence on the particles' size and can be understood by reminding that the value of the entropic potential at the bottlenecks increases with larger particle radius since there are less accessible positions for a larger particle. In other words, the effective boundaries confine

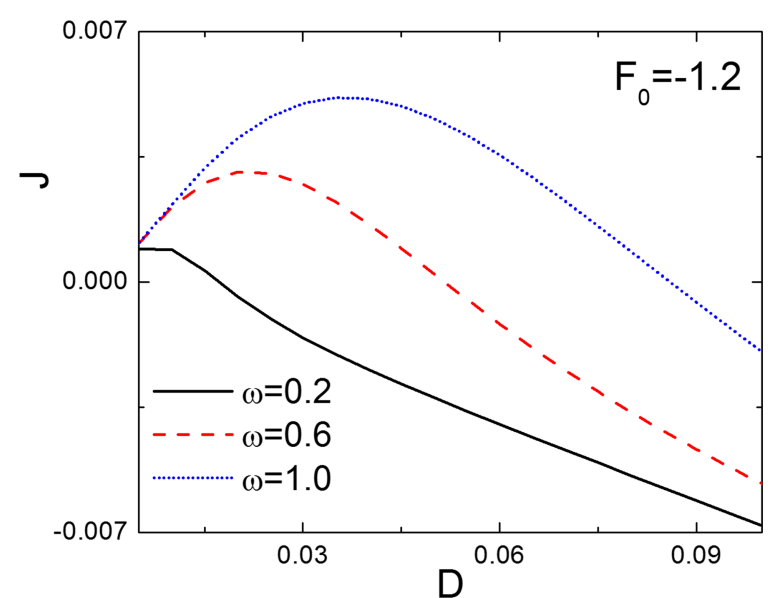

FIG. 5. J versus $D$ for $\omega=0.1$ (black), $\omega=0.5$ (red), and $\omega=0.8$ (blue), and $F_{0}=-1.3$. The values of the remaining parameters are the ones used in Fig. 3. 
larger particles in a stronger way than the boundaries associated with the smaller ones. The large particles are guided to the bottleneck if the external force points to the right, whereas a force that points to the left leads to trapping of the particles at the channel wall if the particles are not located in the area of the bottlenecks. However the interplay between these two effects will depend on the joint action between the external drift and the periodic change in time of the effective boundaries. It is important to note that a uniform distribution in transverse direction is used in the argumentation (Fick-Jacobs validity).

In order to display all these effects together, we show in Fig. 6 the current in the $D-\omega$ plane. In the figure, we indicate the region where current reversal occurs which separates a region of negative monotonic current from a region of positive current with resonant-type behavior. This interesting phenomenon suggests some kind of diffusion-selected effect. Given that the diffusion coefficient $D$ depends on the particle size, by adjusting $\omega$ it is possible to separate particles moving in different directions or in the same direction but with different velocities.

Note that there are different time scales involved. The fastest one is associated with the equilibration in the transversal direction. Another one is associated with the inverse of the frequency of the oscillations and a third one associated with the diffusion process. It is important to keep in mind that although $D$ is constant, the effective diffusion coefficient given in Eq. (4) displays a dependence on the position and time that although weakly affects locally the diffusion time scale. To analyze the relation between the last two scales, we plot in Fig. 7 the average particle current $\mathrm{J}$ as a function of $\omega$ for three different values of $D$ and $F_{0}=-1.2$. We observe that $J$ increases monotonically with $\omega$, regardless of the value of $D$. In the adiabatic limit, the pulsation can be expressed by a boundary in two static configurations. The particles have enough time to explore the whole area of the unit. If $F_{0}=0$, the probability of particles acting on the slanted wall (right part of the subunit) should be larger than that on the steep one (smaller area in the left) resulting in a negative entropic force. However when $F_{0}$ is negative, more particles are located near the steep region resulting in a positive effective entropic force. On increasing $\omega$, the particle does not get enough time to explore

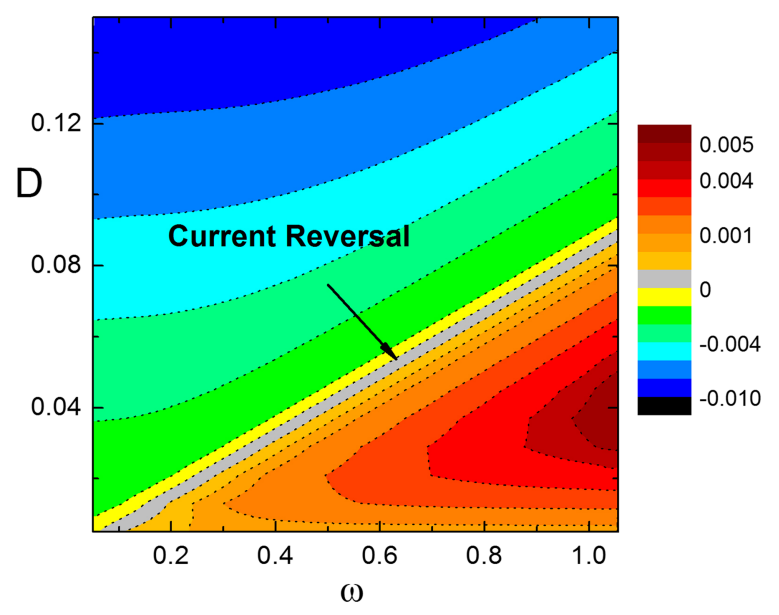

FIG. 6. Contour plot for $\mathrm{J}$ versus $\omega$ and $D . F_{0}=-1.3$ and other parameters as in Fig. 3.

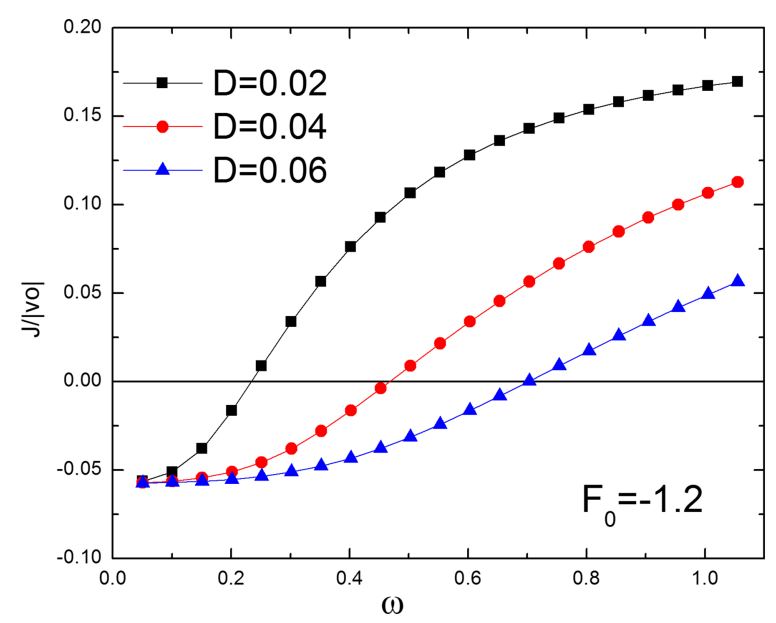

FIG. 7. J versus $\omega$ for $D=0.02$ (black), 0.04 (red), and 0.08 (blue), and $F_{0}$ $=-1.2 \mathrm{~J}$ is normalized by the velocity $v_{0}=\left|D F_{0}\right|$ of the unbounded system.

the full region in one period. The probability density in the area of the steep wall is now larger along one period of time so most particles are subject to the effective (positive) force acting in this region. This effect is enhanced for particles of smaller size. The current is still negative but with a smaller absolute value. Therefore, by controlling (increasing) $\omega$, it is possible to switch to a positive net current. Moreover, the current inversion is observed for greater $\omega$ as long as $D$ increases. The tuning between the diffusion and oscillation time scales is the underlying mechanism.

Motivated by current experimental studies on transport of colloidal particles based on tracking techniques by video microscopy, it is also useful in the complementary analysis of the time evolution of the ensemble average position. In Fig. 8, the mean position of the ensemble of particles (Eq. (10)) versus time is depicted. Initially, particles are located in one unit cell. Fig. 8(a) evidences the expected sensitivity of the current to the strength of the static force. The increase of $\left|F_{0}\right|$ means larger $|J|$ unless $F_{0}$ takes values close to the current inversion. It is also interesting to note the behavior with $D$ shown in Fig. 8(b). For instance, when $F_{0}=-1.2$ (close to CR) and low diffusion, $X(t)$ is confined in one unit cell. When $D$ increases, the motion is mostly bounded during longer times but slightly drifted. For higher diffusion, the motion is still oscillatory but with an important drift. Even more, in one period the oscillations take place between neighbor units consequently with larger amplitudes.

As it was discussed, it is possible to adjust the velocity by just changing the frequency when assuming constant diffusion (Fig. 8(c)). That is, depending on $\omega$, it is possible to rectify in a desired direction even for a fixed static force as it is shown in Fig. 8(c). For instance, for $F_{0}=-1.1$ and low frequency, particles move to the left. However by increasing $\omega$, the mean position is confined in one unit of the channel during many periods with a small drift to the right. Something similar is observed for $F_{0}=-1.3$. Approaching the condition for current inversion, we observe that the oscillatory motion of the center of mass of the ensemble spend many periods inside one unit cell. CR phenomenon corresponds essentially to an oscillatory motion of the center of mass inside one unit cell without a net drift from one side to the other side of the bottleneck. Therefore 
(a)

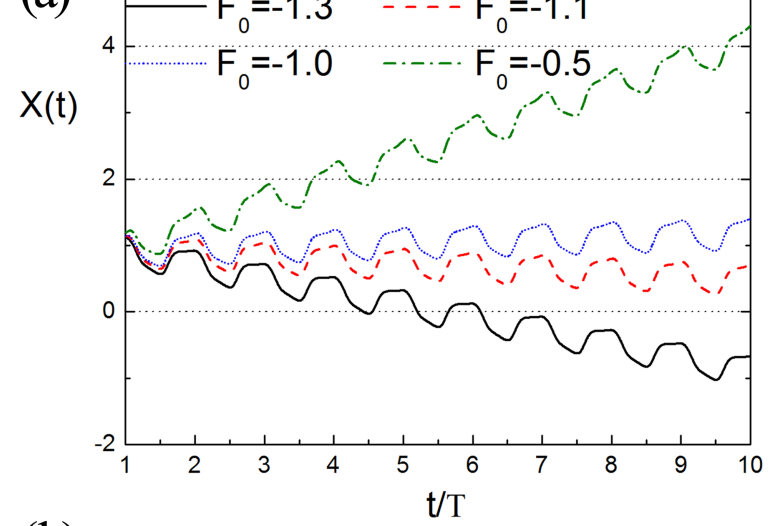

(b)

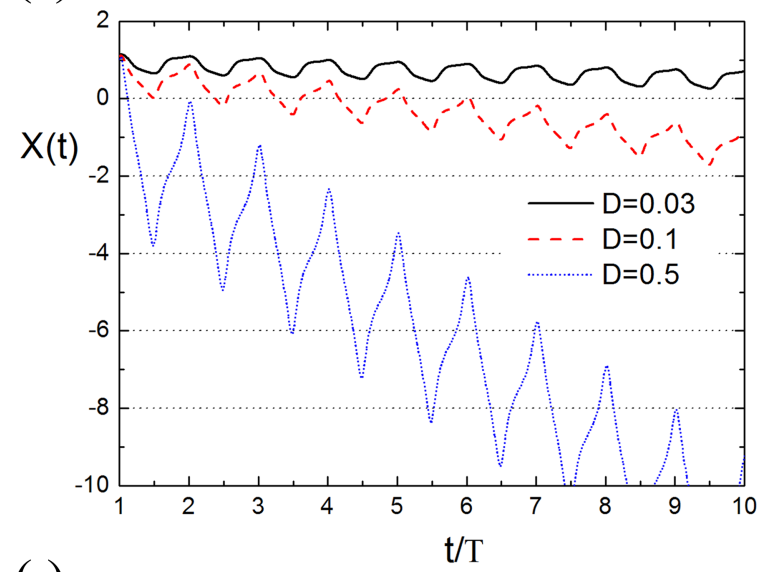

(c)

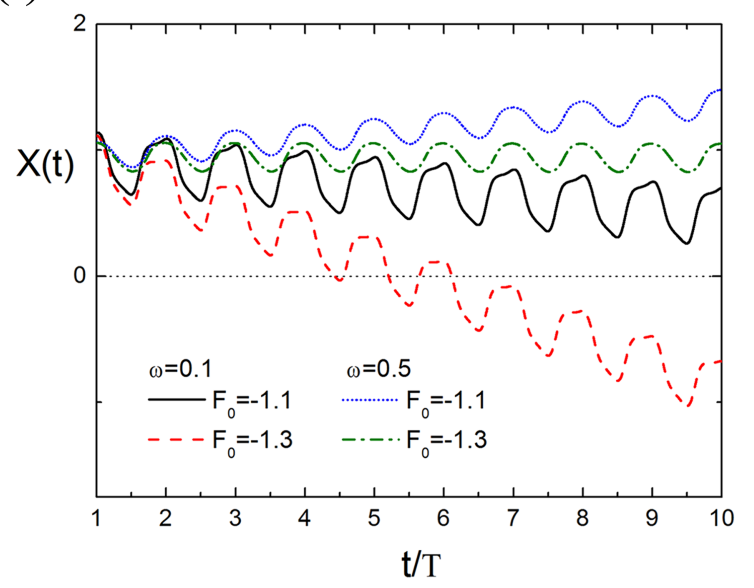

FIG. 8. $X(t)$ versus $t / T$ for different values of $F_{0}$ (a), $D\left(\right.$ b), and $F_{0}$ and $\omega$ (c). Horizontal dot lines separate units of length. The remaining parameters correspond to the ones in Fig. 3.

depending on $F_{0}, D$, and $\omega$, the oscillatory evolution of $X(t)$ can be bounded or unbounded with amplitudes smaller or larger than the length of one unit cell. This mechanism can be used as the basis for a dynamical selective rectifier or a setup sensitive to specific properties of the colloids. In previous works, ${ }^{35,36}$ a particle splitter based on a static geometry under the action of time dependent external forces was studied. However in our model, the central mechanism is more complex because it relies on the modulation of the walls and consequently on an effective time dependent force but also on an effective diffusion that depends on time and position.
The time evolution of the mean position in one period of time is determined by the evolution of the probability density $P(x, t)$. In Fig. 9, we plot $P(x, t)$ at four different times within a period $T$. The figures correspond to $D=0.03$ and $\omega=0.1$ (a) and 0.5 (b) with $F_{0}=-1.3$. The probability distribution oscillates inside one unit cell during a period of time. If $D$ is small, the particles are located more likely in one subunit every half time period, but for greater $D$, in the same half period the particles are distributed more equally in both subunits.

In the previous analysis, we have assumed that the phase difference between consecutive units is $\pi$. This is an oversimplified assumption to keep nearly conserved the total volume of the system as it should be expected in many real systems. However in a modelling work, it is also desirable to analyze the transport through unit cells oscillating with a general phase lag. In Fig. 10, we show $J$ as a function of $\Phi$ in the interval $[0, \pi]$. We observe a current reduction when $\Phi \rightarrow \pi$. Therefore out of phase oscillations seem to reduce J. However, the dependence on $D$ is nontrivial. When the phase lag between adjacent subunits is small, diffusion plays the relevant role during the part of the time period in which the entropic force is reduced almost at the same time in all the units. During those intervals, diffusion dominates and so the net current is then more sensitive to $D$ and $F_{0}$. On the other hand, when $\Phi$ is larger, the decrease of the entropic force occurs alternately between neighboring units counterbalancing the diffusive process (and $F_{0}$ ) with the possibility of current inversions. Therefore, the phase lag tunes a kind of transition between a regime where
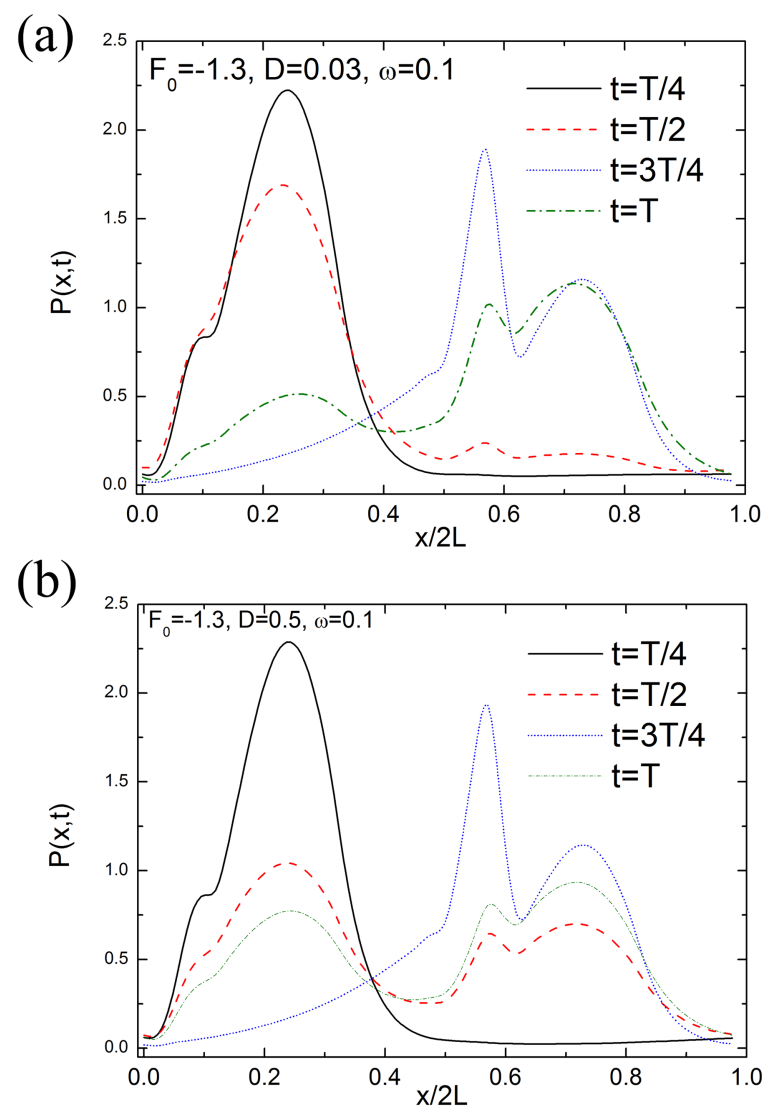

FIG. 9. Probability distribution $P(x, t)$ for $T / 4$ (black full line), $T / 2$ (red dashed line), $3 T / 4$ (blue dot line), and $T$ (green dashed-dot line). $D=0.03$ (a) and $D=0.5$ (b). $F_{0}=-1.3$ and $\omega=0.1$. Lower panel to $D=0.5$. 


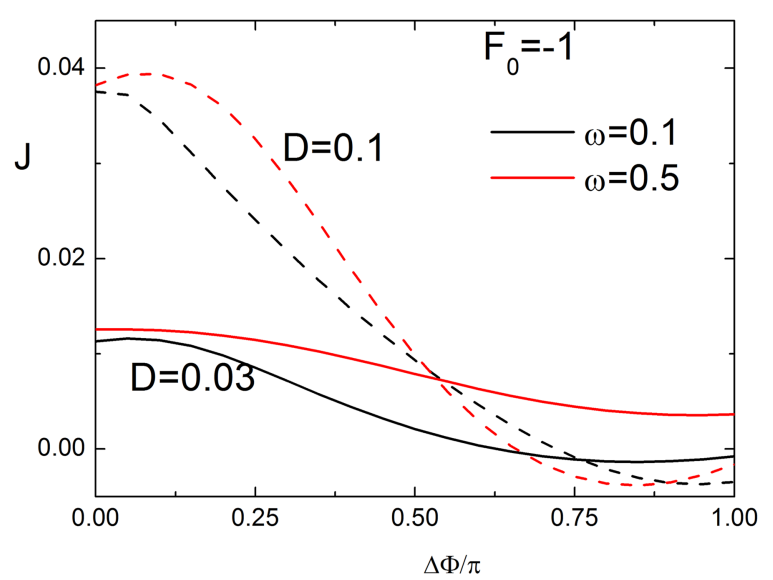

FIG. 10. J versus $\Phi / \pi$ for $D=0.03,0.1$, and $\omega=0.1,0.5 . F=-1.0$.

diffusion is the process that dominates along units during the full period of time, to another one where the oscillation has a comparable relevance.

It is well known that the features of the entropic transport are highly dependent on the asymmetry of the boundaries. The shape and parameters used in our work guarantee an asymmetric geometry meanwhile the walls oscillate. In particular the width of the bottleneck $d$, that is related to the entropic barrier, plays a crucial role related to the flow of particles between adjacent units.

In Fig. 11, we depict a contour plot of $\mathrm{J}$ in the $d-\omega$ plane. For $\omega<0.2$, J increases monotonically with $d$ and for $\omega \approx 0.2$ is almost constant for greater $d$. For $\omega>0.2$, a non-monotonic response with a maximum value of $\mathrm{J}$ that depends on the frequency is obtained. In Fig. 12, we plot the current vs. $d$ for different values of $F_{0}$ and a fixed value of $D$, that is, for particles of a given radius when flowing through channels with bottlenecks of different widths. The three curves have similar shapes; however, they are shifted suggesting an optimal value of $d$ almost independent of $F_{0}$. Moreover, and contrary to what one could expect, a wide bottleneck does not necessarily enhance transport. Also we observe in Fig. 11 that the optimal value of $d$ slightly decreases with $\omega$. Narrower bottlenecks yield the same $J$ when the channel oscillates faster.

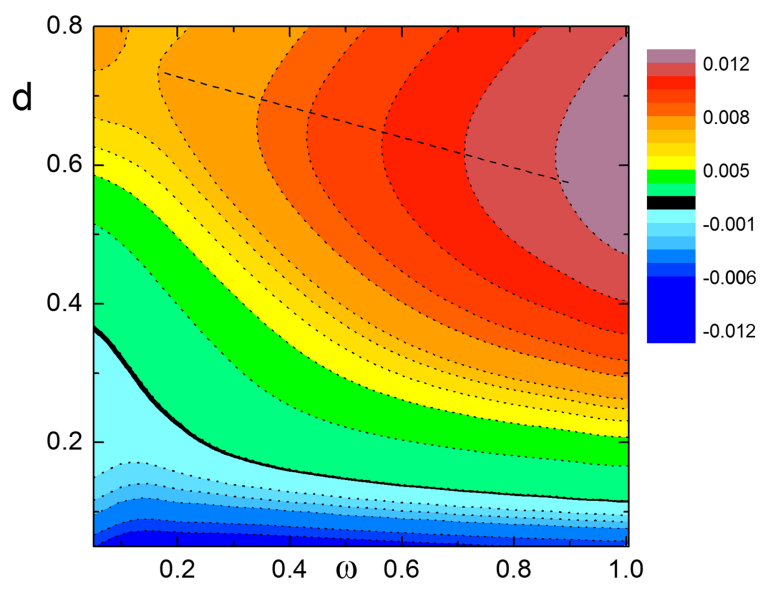

FIG. 11. Average current $\mathrm{J}$ in the $d-\omega$ plane. $F_{0}=-1.1$ and $D=0.03$. The black solid contour line corresponds to current inversion. The dashed line indicates the location of the maximum.

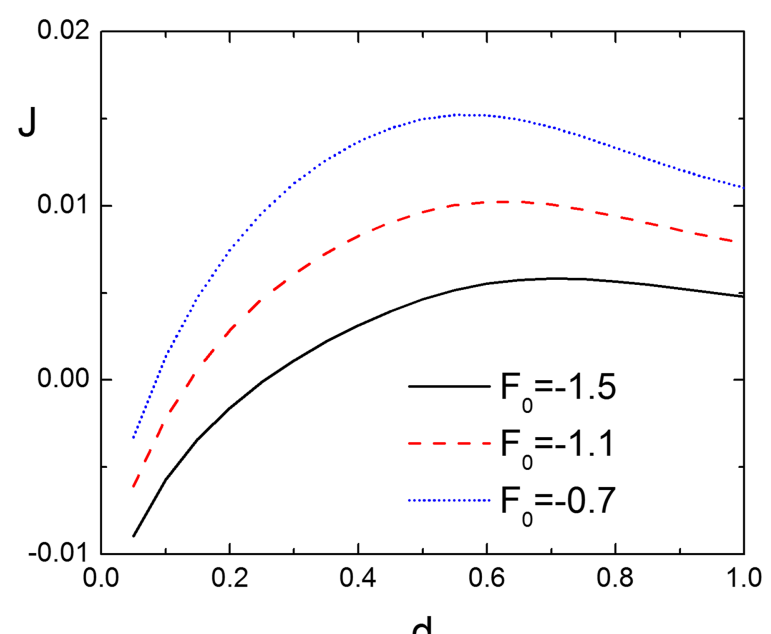

d

FIG. 12. J versus $d$ for $F_{0}=-1.5$ (black full line), -1.1 (red dashed line), and -0.7 (blue dotted line). $D=0.03$ and $\omega=0.5$.

\section{CONCLUSIONS}

We have investigated the dynamics of Brownian particles in a confined channel whose shape is periodically modulated in time. We have identified two distinctive regimes of current rectification. One in the direction of the static external force and another one in an opposite direction, where the entropic contribution plays the key role. The average current presents a monotonic and non-monotonic dependence on $D$ depending on the values of frequencies and external forces. The nonmonotonic case is like a resonant-type response, where the maximum value of $\mathrm{J}$ is strongly dependent on $F_{0}$ and $\omega$. It is opposite to the external force direction and shifted to higher values of $D$ when walls oscillate faster. Moreover, we found a current reversal that takes place when a negative static external force is acting. This phenomenon requires the time-dependent entropic barrier that affects the effective force and diffusion coefficient.

On the other hand, an out of phase oscillation between consecutive units was found to be less beneficial to increase velocity, however it was found to be more favorable for the appearance of CR when $D$ or $F_{0}$ are properly chosen.

Recent works considered the case of directional transport due to time-dependent forces with static ${ }^{37}$ and time-dependent boundaries, constant diffusion, and constant transversal forces. ${ }^{29,30}$ Our model considers a time dependent boundary that has a relevant effect not only on an effective longitudinal force but also on an effective position-time dependent diffusion coefficient. We found that it is possible to rectify the transport but also to optimize and invert its direction, phenomena that appear as a transition between an energy-dominated regime and a time-dependent entropic-dominated one.

Rectification and current reversal obtained from our analysis have also been observed in ratchet devices, ${ }^{38}$ where the directional transport can be achieved through the breaking of temporal and/or spatial symmetry. In our system, the tube oscillations are essential to produce the directional transport that even in the absence of energetic barriers and with a symmetrical shaped tube, a ratcheting effect is possible because the time symmetry is broken. 
Our results show that time-modulated boundaries can play a constructive role in the transport properties of particles. This fact can be interesting in many practical situations as in the rectification of microswimmers motion, Janus particles, substances delivery, or vascular systems, to mention just a few. The pulsating action required could be exerted by externally controlled actions or by the system itself, processes which may be of great significance in physico-chemical or biological systems taking place in confined geometries.

\section{ACKNOWLEDGMENTS}

This work is supported by CONICET-Argentina under Grant No. 14420140100013CO and MICINN of the Spanish Government under Grant No. FIS2015-67837-P.

${ }^{1}$ W. Suginta and M. F. Smith, Phys. Rev. Lett. 110, 238102 (2013).

${ }^{2}$ L. Brun, M. Pastoriza-Gallego, G. Oukhaled, J. Mathé, L. Bacri, L. Auvray, and J. Pelta, Phys. Rev. Lett. 100, 158302 (2008).

${ }^{3}$ C. Kreuter, U. Siems, P. Nielaba, P. Leiderer, and A. Erbe, Eur. Phys. J.: Spec. Top. 22(11), 2923-2939 (2013).

${ }^{4}$ Z. Siwy, I. D. Kosińska, A. Fuliński, and C. R. Martin, Phys. Rev. Lett. 94, 048102 (2005).

${ }^{5}$ P. Malgaretti, I. Pagonabarraga, and J. M. Rubi, J. Chem. Phys. 138, 194906 (2013).

${ }^{6}$ P. Malgaretti, I. Pagonabarraga, and J. M. Rubi, Front. Phys. 1, 21 (2013).

${ }^{7}$ P. Malgaretti, I. Pagonabarraga, and J. M. Rubi, Eur. Phys. J.: Spec. Top. 223(14), 3295-3309 (2014).

${ }^{8}$ L. Liu, P. Li, and S. A. Asher, Nature 397, 141-144 (1999).

${ }^{9}$ R. Zwanzig, J. Phys. Chem. 96, 3926 (1992).

${ }^{10}$ D. Reguera and J. M. Rubí, Phys. Rev. E 64, 061106 (2001).

${ }^{11}$ P. S. Burada, G. Schmid, D. Reguera, J. M. Rubí, and P. Hänggi, Phys. Rev. E 75(5), 051111 (2007).

${ }^{12}$ L. Dagdug, A. M. Berezhkovskii, Y. A. Makhnovskii, V. Y. Zitserman, and S. M. Bezrukov, J. Chem. Phys. 136(21), 214110 (2012).
${ }^{13}$ E. Muñoz-Gutiérrez, J. Alvarez-Ramirez, L. Dagdug, and G. EspinosaParedes, J. Chem. Phys. 136, 114103 (2012).

${ }^{14}$ R. Verdel, L. Dagdug, A. M. Berezhkovskii, and S. M. Bezrukov, J. Chem. Phys. 144, 084106 (2016).

${ }^{15}$ U. Gerland, R. Bundschuh, and T. Hwa, Phys. Biol. 1, 19 (2004).

${ }^{16}$ R. Bundschuh and U. Gerland, Phys. Rev. Lett. 95, 208104 (2005).

${ }^{17}$ U. F. Keyser, B. N. Koeleman, S. Van Dorp, D. Krapf, R. M. M. Smeets, S. G. Lemay, N. H. Dekker, and C. Dekker, Nat. Phys. 2, 473 (2006).

${ }^{18}$ V. Kukla, J. Kornatowski, D. Demuth, I. Girnus et al., Science 272(5262), 702 (1996).

${ }^{19}$ J. Miekisz, J. Gomulkiewicz, and S. Miekisz, Math. Appl. 42(1), 39-62 (2014).

${ }^{20}$ G. M. Makhlouf and K. S. Murthy, Cell. Signalling 9, 269 (1997).

${ }^{21}$ A. Bergner and M. J. Sanderson, J. Gen. Physiol. 119, 187 (2002).

${ }^{22}$ T. J. Moriarty, M. U. Norman, P. Colarusso, T. Bankhead, P. Kubes, and G. Chaconas, PLoS Pathog. 4, 1000090 (2008).

${ }^{23}$ M. A. Dias and T. R. Powers, Phys. Fluids 25, 101901 (2013).

${ }^{24}$ P. Nagarani and G. Sarojamma, Australas. Phys. Eng. Sci. Med. 30(3), 185 (2007).

${ }^{25}$ Y. Mao, S. Chang, S. Yang, Q. Ouyang, and L. Jiang, Nat. Nanotechnol. 2, 366-371 (2007).

${ }^{26}$ P. Hänggi and F. Marchesoni, Rev. Mod. Phys. 81, 387 (2009).

${ }^{27}$ P. Hänggi, F. Marchesoni, and F. Nori, Ann. Phys. 14(1-3), 51 (2005).

${ }^{28}$ P. Kalinay, Phys. Rev. E 84, 011118 (2011).

${ }^{29}$ H. Ding, H. Jiang, and Z. Hou, J. Chem. Phys. 143, 244119 (2015).

${ }^{30}$ H. Ding, H. Jiang, and Z. Hou, J. Chem. Phys. 142, 194109 (2015).

${ }^{31}$ P. Reiman, Phys. Rep. 361, 57 (2002).

${ }^{32}$ D. Lairez, M.-C. Clochard, and J.-E. Wegrowe, Sci. Rep. 6, 38966 (2016).

${ }^{33}$ C. Dalle-Ferrier, M. Krüger, R. D. L. Hanes, S. Walta, M. C. Jenkins, and S. U. Egelhaaf, Soft Matter 7, 2064 (2011).

${ }^{34}$ B. J. Lopez, N. J. Kuwada, E. M. Craig, B. R. Long, and H. Linke, Phys. Rev. Lett. 101, 220601 (2008).

${ }^{35}$ T. Motz, G. Schmid, P. Hänggi, D. Reguera, and J. M. Rubí, J. Chem. Phys. 141, 074104 (2014).

${ }^{36}$ D. Reguera, A. Luque, P. S. Burada, G. Schmid, J. M. Rubí, and P. Hänggi, Phys. Rev. Lett. 108, 020604 (2012).

${ }^{37}$ D. Reguera and J. M. Rubi, Eur. Phys. J.: Spec. Top. 223, 3079 (2014).

${ }^{38}$ R. Bartussek and P. Hänggi, "Nonlinear physics of complex systems," Lect. Notes Phys. 476, 294-308 (2007). 